\title{
A caracterização da assistência que gera violência obstétrica a parturiente: revisão
}

\section{integrativa}

\author{
The characterization of care that generates obstetric violence to parturient women: an integrative \\ review
}

La caracterización del cuidado que genera violencia obstétrica a la parturienta: una revisión

integradora

\author{
Alaíde Régia Araújo Bezerra \\ ORCID: https://orcid.org/0000-0003-2939-7999 \\ Instituto de Ensino Superior Múltiplo, Brasil \\ E-mail: alaideregiaa@gmail.com \\ Anderson Francisco Monteiro da Silva \\ ORCID: https://orcid.org/0000-0002-9142-7493 \\ Instituto de Ensino Superior Múltiplo, Brasil \\ E-mail: anderson_a_fm@hotmail.com \\ Crisnaelle do Nascimento Leal \\ ORCID: https://orcid.org/0000-0001-6288-054X \\ Instituto de Ensino Superior Múltiplo, Brasil \\ E-mail: crisnayele@ hotmail.com \\ Raquel Vilanova Araújo \\ ORCID: https://orcid.org/0000-0002-1752-296X \\ Instituto de Ensino Superior Múltiplo, Brasil \\ E-mail: raquelvilanovabb@gmail.com
}

\begin{abstract}
Resumo
Objetivo: Análise na literatura acerca da caracterização da violência obstétrica sofrida por parturientes na assistência ao parto. Métodos: Trata-se de uma revisão integrativa da literatura, com abordagem qualitativa. Baseada nos materiais disponíveis na Biblioteca Virtual em Saúde. Foram selecionados artigos publicados no período de 2016 a 2021, em texto completo, nas línguas portuguesa, espanhola e inglesa que se relacionavam ao tema proposto. Resultados e discussão: A amostra final constitui-se de 14 artigos, 9 da LILACS, 3 da BDENF e 2 da MEDLINE. Foram analisados e feitos uma leitura minuciosa discutido em relação concordância com o tema apresentado. Para a realização da análise e discussão desta pesquisa inicialmente foi avaliada as seguintes variáveis dos estudos: autores, unidade federativa, ano de publicação, título, revista e base de dados. Agrupados os resultados em eixos temáticos, em três categorias: "A visão da mulher sobre seus direitos no parto"; "Procedimentos invasivos mais recorrentes entre elas"; "Formas de violência sofridas entre as parturientes". Conclusão: O estudo espera contribuir para futuras pesquisas na área da saúde com foco na atuação dos profissionais junto a assistência a parturiente, além de despertar estudantes e profissionais da área a estimular e consequentemente viabilizar mudanças satisfatórias que potencializem a atuação e o crescimento positivo da profissão.
\end{abstract}

Palavras-chave: Violência contra a mulher; Obstetrícia; Assistência ao parto.

\begin{abstract}
Objective: Literature analysis on the characterization of obstetric violence suffered by parturients in childbirth care. Methods: This is an integrative literature review with a qualitative approach. Based on materials available in the Virtual Health Library. Articles published from 2016 to 2021, in full text, in Portuguese, Spanish and English, that related to the proposed theme, were selected. Results and discussion: The final sample consists of 14 articles, 9 from LILACS, 3 from BDENF and 2 from MEDLINE. A thorough reading was analyzed and discussed in accordance with the topic presented. To carry out the analysis and discussion of this research, the following study variables were initially evaluated: authors, federative unit, year of publication, title, journal and database. The results are grouped into thematic axes, in three categories: "The view of women about their rights in childbirth"; "Most recurrent invasive procedures among them"; "Forms of violence suffered among parturient women". Conclusion: The study hopes to contribute to future research in the health area focusing on the performance of professionals with care for parturients, in addition to awakening students and professionals in the area to encourage and consequently enable satisfactory changes that enhance the performance and positive growth of the profession.
\end{abstract}

Keywords: Violence against Women; Obstetrics; Delivery assistance. 


\begin{abstract}
Resumen
Objetivo: Análisis de la literatura sobre la caracterización de la violencia obstétrica que sufren las parturientas en la atención al parto. Métodos: Se trata de una revisión de la literatura integradora con un enfoque cualitativo. Con base en los materiales disponibles en la Biblioteca Virtual en Salud, se seleccionaron los artículos publicados de 2016 a 2021, en texto completo, en portugués, español e inglés, relacionados con el tema propuesto. Resultados y discusión: La muestra final consta de 14 artículos, 9 de LILACS, 3 de BDENF y 2 de MEDLINE. Se analizó y discutió una lectura minuciosa de acuerdo con el tema presentado. Para realizar el análisis y discusión de esta investigación, inicialmente se evaluaron las siguientes variables de estudio: autores, unidad federativa, año de publicación, título, revista y base de datos. Los resultados se agrupan en ejes temáticos, en tres categorías: "La visión de las mujeres sobre sus derechos en el parto"; "Los procedimientos invasivos más recurrentes entre ellos"; "Formas de violencia sufridas entre parturientas". Conclusión: El estudio espera contribuir a futuras investigaciones en el área de la salud enfocadas en el desempeño de los profesionales con el cuidado de parturientas, además de despertar a estudiantes y profesionales del área para incentivar y consecuentemente posibilitar cambios satisfactorios que potencien el desempeño y crecimiento positivo de la profesión.
\end{abstract}

Palabras clave: Violencia contra la mujer; Obstetricia; Asistencia de entrega.

\title{
1. Introdução
}

A partir do século XX, diante de situações no momento do parto, classificadas como de alto risco à mãe e ao bebê, fez com que o uso de tecnologias durante a assistência fosse requerido, institucionalizando assim o mesmo (Cardoso, et al., 2017). A medida em que as práticas intervencionistas são supervalorizadas e hierarquizadas, marcantes dimensões da tecnologia científica são apresentadas, fazendo assim com que o cenário brasileiro atual da assistência ao parto se torne complexo. O mau uso de tecnologias disponíveis e a desvalorização das evidências científicas existentes, gerou práticas nocivas na assistência às gestantes, contribuindo assim para o aumento das taxas de morbimortalidade tanto materna quanto perinatal (Barbosa \& Fabbro \& Machado, 2017).

As mulheres em sua totalidade possuem o direito de saúde de qualidade, como a uma assistência digna durante todo o período da gravidez e parto, estar livre da violência, discriminação, falta de assistência que denigre os direitos humanos internacionalmente. O modelo tecnocrático hegemônico no setor de saúde brasileiro delineou características peculiares à assistência ao parto e nascimento no país, desconsiderando ou negando os desconfortos e possíveis efeitos adversos a elas ligados. Esse cenário favorece a prática da cesárea de rotina, a violação dos direitos da mulher e a manutenção dos elevados números de mortalidade materna. Existem vários quadros perturbadores em mulheres durante seu parto. Como, abusos, desrespeito, maus-tratos e negligência durante a assistência em instituições de saúde. Isso causa uma falta de confiança entre as mulheres e os profissionais de saúde e grande desestímulo das mulheres procurarem serviços de obstetrícia. Embora vários atos aconteçam no parto, esses eventos também têm ocorrências em vários períodos da gravidez (OMS, 2014).

O conceito de violência obstétrica, surge dentro deste contexto. A mesma é expressa pela falta de assistência digna, discriminação socioeconômica e racial, violência verbal, física e psicológica, considerando também ato de violência obstétrica, o uso inadequado de tecnologias e a adoção de procedimentos durante o ciclo gravídico e pós-parto sem o consentimento da gestante, ferindo os direitos individuais da mulher. Esses atos de violência podem ser cometidos por pessoas próximas, desconhecidas, profissionais ou por instituições, contribuem para complicações ou efeitos irreparáveis ao binômio mãe-filho (Andrade, 2016).

\section{Metodologia}

O presente estudo trata-se de uma revisão da literatura integrativa realizada por intermédio de base de dados, que auxiliam na busca de artigos e materiais de conteúdo científico que permite a incorporação de evidências na prática clínica (Sousa, et al., 2017), com abordagem qualitativa, que relata análise na literatura a caracterização da violência obstétrica sofrida por parturientes na assistência ao parto. 
A pesquisa bibliográfica foi realizada usando materiais disponíveis na Biblioteca Virtual em Saúde, nas bases de dados Sistema Online de Busca e Análise de Literatura Médica (MEDLINE), Literatura Latino-Americana e do Caribe em Ciências da Saúde (LILACS), Base de Dados de Enfermagem (BDENF). Utilizaram-se os seguintes descritores na estratégia de busca: violência contra a mulher, obstetrícia, assistência ao parto.

Para uma busca mais abrangente e significativa, os descritores controlados foram combinados de inúmeras maneiras, utilizando os operadores booleanos OR e AND, aplicando somente os Descritores em Ciência da Saúde (Decs) para elaboração da estratégia de busca no portal da biblioteca virtual em saúde (BVS), da seguinte forma: (tw:(Violência obstétrica)) AND (tw: (Obstetrícia)) OR (tw:(Assistência ao Parto)).

Com base na coleta de dados, foram encontrados 6656 estudos. Após a análise, ocorreu a primeira fase, que teve como objetivo à aplicação dos critérios de inclusão e exclusão. Foram incluídos: artigos em texto completo disponíveis de forma gratuita, idiomas português, inglês e espanhol, publicados entre 2016 e 2021. Foram excluídos: artigos duplicados, escrito em outras línguas, que fugiam ao tema proposto e ao período de publicação. Dessa maneira, obteve-se 61 artigos no final da primeira fase. Na segunda fase, realizou-se a leitura dos 61 estudos para detectar aqueles que respondiam e/ou tinham concordância com o tema apresentado. A figura 1 mostra o fluxograma das etapas.

Figura 1 - Fluxograma das etapas de estratégia de busca dos artigos.

\begin{tabular}{l}
\hline ESTRATÉGIA DE BUSCA \\
\begin{tabular}{|l|l|l|l|l|}
\hline COLETA DE DADOS & IBEC & $\begin{array}{l}\text { Coleciona } \\
\text { SUS }\end{array}$ \\
\hline TOTAL DE 61 ARTIGOS ENCONTRADOS & & 01 \\
\hline LILACS & BDENF & MEDLINE & 01 & 01 \\
\hline 28 & 18 & 13 & & \\
\hline
\end{tabular}
\end{tabular}

Após critérios de inclusão e exclusão $=43$ artigos

\begin{tabular}{|l|l|l|l|l|}
\hline LILACS & BDENF & MEDLINE & IBEC & $\begin{array}{l}\text { Coleciona } \\
\text { SUS }\end{array}$ \\
\hline 21 & 15 & 06 & 00 & 01 \\
\hline
\end{tabular}

Análise na literatura sobre a caracterização violência obstétrica sofrida por parturientes na assistência ao parto.

\begin{tabular}{|l|l|l|l|l|}
\hline LILACS & BDENF & MEDLINE & IBEC & $\begin{array}{l}\text { Coleciona } \\
\text { SUS }\end{array}$ \\
\hline 9 & 03 & 02 & 00 & 0 \\
\hline \multicolumn{2}{|l}{ TOTAL: 14 ARTIGOS }
\end{tabular}

Fonte: Dados da pesquisa (2021). 
A partir do fluxograma, resultou-se uma amostra de 14 artigos inclusos na pesquisa, sendo 9 da LILACS, 3 da BDENF e 2 da MEDLINE. A análise e interpretação dos dados foram estruturados nos resultados e discussões do presente artigo.

\section{Resultados e Discussão}

Quadro 1 - Características dos estudos. Teresina - PI, 2021.

\begin{tabular}{|c|c|c|c|c|c|}
\hline ANO & $\begin{array}{c}\text { UF / PAÍS DE } \\
\text { ORIGEM }\end{array}$ & AUTORES & TÍTULO & REVISTA & $\begin{array}{c}\text { BASE DE } \\
\text { DADOS }\end{array}$ \\
\hline
\end{tabular}

\begin{tabular}{|c|c|c|c|c|c|}
\hline 2017 & $\begin{array}{l}\text { California - } \\
\text { USA }\end{array}$ & $\begin{array}{c}\text { Kujawski, } \\
\text { Stephanie A. et al. }\end{array}$ & $\begin{array}{l}\text { Community and health system } \\
\text { intervention to reduce disrespect } \\
\text { and abuse during childbirth in } \\
\text { Tanga region, Tanzania: a } \\
\text { comparative before-and-after } \\
\text { study. }\end{array}$ & Plos Medicine. & MEDLINE \\
\hline 2020 & Oslo - Noruega & $\begin{array}{c}\text { garnweidner- } \\
\text { Holme, Lisa. et al. }\end{array}$ & $\begin{array}{l}\text { Midwives' Attitudes Toward and } \\
\text { Experience With a Tablet } \\
\text { Intervention to Promote Safety } \\
\text { Behaviors for Pregnant Women } \\
\text { Reporting Intimate Partner } \\
\text { Violence: Qualitative Study. }\end{array}$ & $\begin{array}{l}\text { Jmir Mhealth And } \\
\text { Uhealth }\end{array}$ & MEDLINE \\
\hline 2016 & RJ - Brasil & $\begin{array}{l}\text { Rodrigues, Diego } \\
\text { Pereira } \text { et al. }\end{array}$ & $\begin{array}{l}\text { Violência obstétrica no processo } \\
\text { do parto e nascimento da região } \\
\text { metropolitana II do Estado do Rio } \\
\text { de Janeiro: percepção de } \\
\text { mulheres/puérperas. }\end{array}$ & $\begin{array}{l}\text { PACCS - Teses E } \\
\text { Dissertações }\end{array}$ & LILACS \\
\hline 2016 & PE - Brasil & $\begin{array}{c}\text { De Oliveira } \\
\text { Nascimento } \\
\text { Andrade, Priscyla } \\
\text { et al. }\end{array}$ & $\begin{array}{l}\text { Fatores associados à violência } \\
\text { obstétrica na assistência ao parto } \\
\text { vaginal em uma maternidade de } \\
\text { alta complexidade em Recife, } \\
\text { Pernambuco. }\end{array}$ & $\begin{array}{c}\text { Revista Brasileira } \\
\text { De Saude Materno } \\
\text { Infantil, }\end{array}$ & LILACS \\
\hline 2016 & MT - Brasil & $\begin{array}{l}\text { Knupp Medeiros, } \\
\text { Renata Marien } e t \\
\text { al. }\end{array}$ & 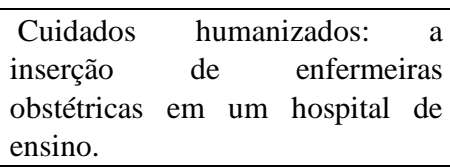 & $\begin{array}{l}\text { Revista Brasileira } \\
\text { De Enfermagem }\end{array}$ & LILACS \\
\hline 2016 & RS - Brasil & $\begin{array}{l}\text { Brüggemann, } \\
\text { Odaléa Maria } e t \\
\text { al. }\end{array}$ & $\begin{array}{l}\text { No parto vaginal e na cesariana } \\
\text { acompanhante não entra: } \\
\text { discursos de enfermeiras e } \\
\text { diretores técnicos. }\end{array}$ & $\begin{array}{l}\text { Revista Gaúcha } \\
\text { De Enfermagem }\end{array}$ & LILACS \\
\hline 2016 & RJ- Brasil & $\begin{array}{c}\text { Vieira, Bianca } \\
\text { Dargam Gomes et } \\
\text { al. }\end{array}$ & $\begin{array}{l}\text { As implicações da prática } \\
\text { profissional de enfermeiros } \\
\text { obstetras egressos da EEAN: a } \\
\text { qualidade da assistência. }\end{array}$ & $\begin{array}{c}\text { Revista De } \\
\text { Pesquisa: Cuidado } \\
\text { É Fundamental } \\
\text { Online. }\end{array}$ & LILACS \\
\hline 2016 & $\begin{array}{c}\text { Caracas - } \\
\text { Venezuela }\end{array}$ & Terán, Pablo et al. & $\begin{array}{l}\text { Violencia obstétrica: percepción } \\
\text { de las usuarias. }\end{array}$ & $\begin{array}{c}\text { Revista De } \\
\text { Obstetricia Y } \\
\text { Ginecología De } \\
\text { Venezuela. }\end{array}$ & LILACS \\
\hline 2016 & $\begin{array}{c}\text { Caracas } \\
\text { Venezuela }\end{array}$ & $\begin{array}{l}\text { Pereira, Carlota; } \\
\text { Domínguez, } \\
\text { Alexa; Toro, } \\
\text { Judith. }\end{array}$ & $\begin{array}{l}\text { Violencia obstétrica desde la } \\
\text { perspectiva de la paciente. }\end{array}$ & $\begin{array}{c}\text { Revista De } \\
\text { Obstetricia Y } \\
\text { Ginecología De } \\
\text { Venezuela. }\end{array}$ & LILACS \\
\hline
\end{tabular}




\begin{tabular}{|c|c|c|l|c|c|}
\hline 2016 & SC - Brasil & $\begin{array}{c}\text { Tesser, Charles } \\
\text { Dalcanale et al. }\end{array}$ & $\begin{array}{l}\text { Violência obstétrica e prevenção } \\
\text { quaternária: o que é e o que fazer. }\end{array}$ & $\begin{array}{c}\text { Revista Brasileira } \\
\text { De Medicina De } \\
\text { Família E } \\
\text { Comunidade. }\end{array}$ & LILACS \\
\hline 2019 & SP - Brasil & $\begin{array}{c}\text { Diniz, Simone } \\
\text { Grilo et al }\end{array}$ & $\begin{array}{l}\text { Violência obstétrica como questão } \\
\text { para a saúde pública no Brasil: } \\
\text { origens, definições, tipologia, } \\
\text { impactos sobre a saúde materna, e } \\
\text { propostas para sua prevenção. }\end{array}$ & $\begin{array}{c}\text { Journal Of Human } \\
\text { Growth And } \\
\text { Development. }\end{array}$ & LILACS \\
\hline 2017 & MG - Brasil & $\begin{array}{c}\text { Ferreira, Lúcia } \\
\text { Aparecida et al. }\end{array}$ & $\begin{array}{l}\text { Expectativa das gestantes em } \\
\text { relação ao parto. }\end{array}$ & $\begin{array}{c}\text { Rev. Pesqui. } \\
\text { Cuid. Fundam } \\
\text { (Online). }\end{array}$ & BDENF \\
\hline 2017 & PE- Brasil & $\begin{array}{c}\text { Oliveira, Mayra de } \\
\text { Castro; Merces, } \\
\text { Magno Conceição } \\
\text { das. }\end{array}$ & $\begin{array}{l}\text { Percepções sobre violências } \\
\text { obstétricas na ótica de puérperas. }\end{array}$ & $\begin{array}{l}\text { Rev. Enferm. } \\
\text { Ufpe Online. }\end{array}$ & BDENF \\
\hline 2017 & PE- Brasil & $\begin{array}{c}\text { Cardoso, Ferdinand } \\
\text { José da Costa et al. }\end{array}$ & $\begin{array}{l}\text { Violência obstétrica institucional } \\
\text { no parto: percepção de } \\
\text { profissionais da saúde. }\end{array}$ & $\begin{array}{c}\text { Rev. Enferm. } \\
\text { Ufpe On Line. }\end{array}$ & BDENF \\
\hline
\end{tabular}

Fonte: Pesquisa direta em base de dados.

Foi possível observar que as unidades federativas com mais estudos relacionados a tal assunto, foram São Paulo e Rio de Janeiro, prevalecendo a região sudeste do Brasil com maior desenvolvimento de artigos sobre o tema. Foi possível verificar ainda, que a maioria das pesquisas aponta que os profissionais de saúde se expõem diretamente em condições de violência obstétrica, acompanhantes e as parturientes. Constatou-se ainda que os principais fatores de violências são: proibição de acompanhantes, métodos precoces para a aceleração do parto, e torturas verbais e psicológicas.

O presente estudo procurou compilar a produção científica mais atual acerca violência obstétrica. Para a realização da análise e discussão desta pesquisa inicialmente foi avaliada as seguintes variáveis dos estudos: autores, unidade federativa/país de origem, ano de publicação, título, revista e base de dados e posteriormente também foi possível agrupar os resultados em eixos temáticos, onde suscitaram três categorias, a saber: "A visão da mulher sobre seus direitos no parto"; "Procedimentos invasivos mais recorrentes entre elas"; "Formas de violência sofridas entre as parturientes".

\section{A visão da mulher sobre seus direitos no parto}

Na transformação da situação de mulher para o de mãe, o parto é um momento crucial de construção da identidade materna. O parto como uma cerimônia transformativa apresenta uma ampla dessemelhança social, de acordo com culturas, religiões, etnias e classe social. Nessa direção, identificou-se que as circunstâncias e expectativas das mulheres são partes características da experiência do parto. Nos relatos das parturientes elas assimilam a episiotomia como uma forma de alivio à agonia; como uma viabilidade de diminuir o tempo de expulsão do feto, porém diversas não sabem dos riscos do procedimento ou até desconhecem, principalmente quando se comunicam com a parturiente de forma técnica, usando termos científicos quando se trata de uma paciente leiga no assunto (Rodrigues, et al., 2016).

No entanto, demonstrou-se desconhecimento sobre suas indicações, evidenciando uma distorção da visão das mulheres, por um tipo de impregnação do modelo assistencial a saúde ou tecnocrático. Este tipo de deturpação é reforçado pela falta de autonomia das mulheres no processo do parto e do nascimento. Entende-se que se torna urgente a inclusão do respeito aos direitos humanos das mulheres, sublinhando-se os direitos sexuais e reprodutivos na perspectiva da promoção da saúde, no âmbito da sexualidade e reprodução (Oliveira \& Merces, 2017).

O corpo da mulher passa a ser responsabilidade da equipe multiprofissional, permitindo-lhes pensar que isso era feito como instrumento de controle social. O domínio exercido sobre o corpo feminino relaciona-se ao aperfeiçoamento de uma 
tecnologia que permitiu a resolução dos problemas cruciais para a sobrevivência das mulheres e crianças, aflorando num momento em que a conservação da vitalidade e da saúde é essencial para a reprodução social. Este controle surge da prática profissional determinante do tipo de parto, da hora do parto e do nascimento e, também, do uso de medicação para acelerar as contrações (Valadão \& Pegoraro, 2020).

Ao serem questionadas sobre como o profissional deve tratar as mulheres durante o momento do parto, as mulheres citam a importância de um tratamento digno, atencioso, acolhedor e sintonizado com as suas necessidades. Atentar ao que elas têm a dizer, ter serenidade com as suas dores, passar bonança informando-as do que está acontecendo e salientando o que deve ser e está sendo feito são solicitações importantes dessas mulheres frente às experiências de violência institucional, como piadas, discriminações e intolerância (Rodrigues, et al., 2016).

\section{Procedimentos invasivos mais recorrentes entre elas}

Segundo Zanardo, et al., (2017), há uma necessidade de propiciar um ambiente de saúde que seja mais adequado tanto às gestantes como aos profissionais, pois eles acabam prestando uma assistência de acordo com à sua experiência e as ferramentas disponíveis na instituição, que muitas vezes são insuficientes e as gestantes acabam se submetendo às práticas assistências desnecessárias que podem trazer danos à sua saúde.

A má assistência à gestante no Brasil, o modelo intervencionista ainda é persistente, pois as parturientes ainda estão submetidas às práticas prejudiciais como a episiotomia, que aumenta o risco de infecção e hemorragias, o uso de ocitocina utilizado de maneira equívoca, manobra de Kristeller, além de exames vaginais rotineiros e repetitivos sem justificativa, esses são os procedimentos mais recorrentes utilizados entre as parturientes e que geram violência obstétrica (Oliveira \& Nascimento \& Andrade, 2016).

De acordo com Brüggemann, et al., (2015), a presença de um acompanhante no momento do parto evitaria intervenções desnecessárias durante a assistência, tanto obstétrica como neonatal, que resultam negativamente nos índices da saúde. Esse acompanhamento possibilitaria a mulher receber um aparato maior de apoio social, segurança, além de contribuir para melhores desfechos maternos e neonatais.

A presença de acompanhante de escolha da mulher, durante o período de trabalho de parto, parto e pós-parto imediato, foi regulamentada no Brasil, por meio da Lei ${ }^{\circ}$ 11.108, de 7 de abril de 2005. Esta medida é reafirmada pela Portaria GM/MS $\mathrm{n}^{\circ}$ 1.459, de 24 de junho de 2011, que institui a Rede Cegonha no âmbito do Sistema Único de Saúde (SUS) com o propósito de garantir parto e nascimento seguros às mulheres e seus recém-nascidos, a partir da presença de acompanhante de sua livre escolha. A Organização Mundial da Saúde (OMS) recomenda a presença de acompanhante de livre escolha da gestante como uma das ações de boas práticas de atenção ao parto e nascimento, com vistas à redução de intervenções desnecessárias e violências obstétricas (Tomasi, 2021).

\section{Formas de violência sofridas entre as parturientes}

A proibição de acompanhantes homens na sala de pré-parto, sob a alegação de falta de espaço físico que garanta a privacidade para as demais pacientes é uma das reclamações que as pacientes tem, além dos profissionais de saúde designarem sinônimos a elas, como "escandalosas", ou "a não colaborativa". Por muitas vezes os profissionais que exercem essa função confundem com o exercício da autoridade em um contexto "difícil" (Medeiros \& Martins \& Camboim, 2016).

Frequentemente em centros de parto encontram-se mulheres seminuas na presença de estranhos, sozinhas em um cenário desconhecido, em posição de submissão total devido estarem em cuidados obstétricos, pernas abertas e levantadas, genitália exposta, rotineiramente separadas de seus filhos logo após o nascimento. $O$ conceito estereotipado machista de gênero socialmente difundido, em que a mulher, vista como sexo frágil, necessita ser mantida sob uma autoridade patriarcal (nesse 
cenário, o profissional do sexo masculino), que vai decidir o que é melhor para ela, transformando o nascer em um ato centrado no profissional e passível de prováveis práticas violentas (Jardim \& Modena, 2018).

Para Sena e Tesser (2016), a violência institucional é mascarada, invisível, decorrente de fatores como: dificuldade do usuário em criticar o serviço público de saúde e os profissionais que fizeram seu atendimento, aceitação, banalização do desrespeito visto como "brincadeira" e no caso da assistência perinatal em que a mãe se sente abrandecida e grata pelo nascimento do bebê, acreditando que neutraliza qualquer ato de maus-tratos.

Condutas violentas como o uso de palavras pejorativas, ameaças e reprimendas contra as pacientes no cotidiano da assistência em maternidades e negligência no manejo da dor, em alguns locais, são comuns e até consensuais entre os profissionais. Queixas sobre a postura de profissionais de saúde de não fornecer informações quanto ao estado clínico de seus bebês, especialmente quando são prematuros e levados à UTI, onde também não foi permitida sua participação nos cuidados os gerais do menor (Diniz, et al. 2019).

Violar os direitos dessas mulheres a um atendimento de qualidade, colocar em risco a sua integridade física e mental em um momento de extrema singularidade. Sendo assim, além de um problema de saúde pública, tem-se uma questão de direitos humanos. A violência, seja de ordem física, emocional ou simbólica, é produtora de elevado grau de sofrimento sendo, por vezes, apresentada de forma tão sútil que chega a ser difícil enxergá-la e, consequentemente, dar maior notoriedade a temática (Carvalho \& Brito, 2017).

\section{Conclusão}

Esta pesquisa levanta a reflexão sobre a questão da caracterização da assistência que gera violência obstétrica, evidenciou-se algumas lacunas no que se diz respeito as ações do ato de violência, em destaque as relacionadas a educação em saúde, informações dadas aos profissionais afim da mudança de comportamento, orientações de procedimentos, contribuindo para dependência da mulher ao serviço de saúde, sem abertura para discussão e troca de experiencias.

Diante disso, este estudo espera contribuir para futuras pesquisas na área da saúde com foco na atuação dos profissionais junto a assistência a parturiente, além de despertar estudantes e profissionais da área a estimular e consequentemente viabilizar mudanças satisfatórias que potencializem a atuação e o crescimento positivo da profissão, desenvolvendo a autonomia regada a capacitação e especialização, aumentando a participação efetiva para um cuidados digno e sem violação de todos os direitos que a mulher possa ter em todos os âmbitos do cuidado.

Os resultados encontrados nesta revisão integrativa expressam a necessidade de promover um entorno de saúde mais adequado tanto para as usuárias como para os profissionais, no qual os procedimentos sejam mais regularizados, claros e organizados, e propiciem um ambiente mais seguro. Por isso, mudanças nas práticas assistenciais vigentes devem ser feitas para reduzir as intervenções desnecessárias.

\section{Referências}

Almeida, M. M., da Costa Cardoso, F. J., da Costa, A. C. M., dos Santos, T. S., \& Oliveira, F. B. M. (2017). Violência obstétrica institucional no parto: percepção de profissionais da saúde. Revista de Enfermagem UFPE on line, 11(9), 3346-3353.7

Almeida, M. M., da Costa Cardoso, F. J., da Costa, A. C. M., dos Santos, T. S., \& Oliveira, F. B. M. (2017). Violência obstétrica institucional no parto: percepção de profissionais da saúde. Revista de Enfermagem UFPE on line, 11(9), 3346-3353.

Andrade, P. D. O. N., Silva, J. Q. P. D., Diniz, C. M. M., \& Caminha, M. D. F. C. (2016). Fatores associados à violência obstétrica na assistência ao parto vaginal em uma maternidade de alta complexidade em Recife, Pernambuco. Revista Brasileira de Saúde Materno Infantil, $16,29-37$.

Andrade, P. D. O. N., Silva, J. Q. P. D., Diniz, C. M. M., \& Caminha, M. D. F. C. (2016). Fatores associados à violência obstétrica na assistência ao parto vaginal em uma maternidade de alta complexidade em Recife, Pernambuco. Revista Brasileira de Saúde Materno Infantil, $16,29-37$.

Barbosa, L. D. C., Fabbro, M. R. C., \& Machado, G. P. D. R. (2017). Violência obstétrica: revisão integrativa de pesquisas qualitativas. Avances en Enfermería 
Barbosa, L. D. C., Fabbro, M. R. C., \& Machado, G. P. D. R. (2017). Violência obstétrica: revisão integrativa de pesquisas qualitativas. Avances en Enfermería.

Brüggemann, O. M., Ebele, R. R., Ebsen, E. S., \& Batista, B. D. (2015). No parto vaginal e na cesariana acompanhante não entra: discursos de enfermeiras e diretores técnicos. Revista Gaúcha de Enfermagem, 36, 152-158.

da Silva Carvalho, I., \& De Brito, R. S. (2017). Formas de violência obstétrica vivenciadas por puérperas que tiveram parto normal. Enfermería Global, 16(3), 71-97.

de Medeiros, N. C. M., Martins, E. N. X., \& de Farias Camboim, F. E. (2016). Violência obstétrica: percepções acerca do parto normal.

Diniz, S. G., Salgado, H. D. O., Andrezzo, H. F. D. A., Carvalho, P. G. C. D., Carvalho, P. C. A., Aguiar, C. D. A., \& Niy, D. Y. (2015). Violência obstétrica como questão para a saúde pública no Brasil: origens, definições, tipologia, impactos sobre a saúde materna, e propostas para sua prevenção. $J$ Hum Growth Dev, 25(3), 377-82.

Ferreira, L. A., Zuffi, F. B., Mauzalto, A. C. M., da Penha Leite, C., \& Nunes, J. S. (2013). Expectativa de gestantes em relação ao parto / expectativa das gestantes em relação ao parto / expectativa de la mujer embarazada en relación al parto. Revista de Pesquisa, Cuidado é Fundamental Online, 5 (2), 3692.

Garnweidner-Holme, L., Henriksen, L., Flaathen, E. M, Bøhler, T. K., \& Lukasse, M. (2020). Atitudes e experiência das parteiras em relação a uma intervenção do tablet para promover comportamentos de segurança para mulheres grávidas que relatam violência por parceiro íntimo: estudo qualitativo. JMIR mHealth e uHealth, 8 (5), e16828

Jardim, D. M. B., \& Modena, C. M (2018). A violência obstétrica no cotidiano do atendimento e suas características. Revista latino-americana de enfermagem, 26 .

Kujawski, S. A., Freedman, L. P., Ramsey, K., Mbaruku, G., Mbuyita, S., Moyo, W., \& Kruk, M. E. (2017). Intervenção da comunidade e do sistema de saúde para reduzir o desrespeito e o abuso durante o parto na região de Tanga, Tanzânia: um estudo comparativo antes e depois. Medicina PLoS , 14 (7), e1002341.

Medeiros, R. M. K., Teixeira, R. C., Nicolini, A. B., Alvares, A. S., Corrêa, Á. C. D. P., \& Martins, D. P. (2016). Cuidados humanizados: a inserção de enfermeiras obstétricas em um hospital de ensino. Revista Brasileira de Enfermagem, 69, 1091-1098.

Oliveira, M. D. C., \& Merces, M. C. D. (2017). Percepções sobre violências obstétricas na ótica de puérperas. Rev. enferm. UFPE on line, $2483-2489$.

Organização Mundial de Saúde. (2014). Prevenção e eliminação de abusos, desrespeito e maus-tratos durante o parto em instituições de saúde.

Pereira, C., Domínguez, A., \& Toro, J. (2015). Violencia obstétrica desde la perspectiva de la paciente. Revista de Obstetricia y Ginecología de Venezuela, 75(2), 081-090.

Rodrigues, D. P. (2014). Violência obstétrica no processo do parto e nascimento da região metropolitana II do Estado do Rio de Janeiro: percepção de mulheres/puérperas.

Terán, P., Castellanos, C., González Blanco, M., \& Ramos, D. (2013). Violencia obstétrica: percepción de las usuarias. Revista de Obstetricia y Ginecología de Venezuela, 73(3), 171-180.

Tesser, C. D., Knobel, R., de Aguiar Andrezzo, H. F., \& Diniz, S. G. (2015). Violência obstétrica e prevenção quaternária: o que é e o que fazer. Revista Brasileira de Medicina de Família e Comunidade, 10(35), 1-12.

Tomasi, Y. T., Saraiva, S. D. S., Boing, A. C., Delziovo, C. R., Wagner, K. J. P., \& Boing, A. F. (2021). Do pré-natal ao parto: um estudo transversal sobre a influência do acompanhante nas boas práticas obstétricas no Sistema Único de Saúde em Santa Catarina, 2019. Epidemiologia e Serviços de Saúde, 30.

Valadão, C. L., \& Pegoraro, R. F. (2020). Vivências de mulheres sobre o parto. Fractal: Revista de Psicologia, 32 , 91-98.

Vieira, B. D. G., Moura, M. A. V., Alves, V. H., \& Rodrigues, D. P. (2013). As implicações da prática profissional de enfermeiros obstetras egressos da EEAN: a qualidade da assistência. Revista de Pesquisa: Cuidado é fundamental online, 5(4), 408-416. 